\title{
Effect of pre and Post-Dispersion \\ on Electro-Thermo-Mechanical Properties of a Graphene Enhanced Epoxy
}

\author{
Quentin-Arthur Poutrel ${ }^{1} \cdot$ Zixin Wang $^{1} \cdot$ \\ Dongyi Wang ${ }^{1} \cdot$ Constantinos Soutis $^{2}$. \\ Matthieu Gresil ${ }^{1,2}$ (D)
}

Received: 26 September 2016 / Accepted: 3 October 2016 / Published online: 19 October 2016

(C) The Author(s) 2017. This article is published with open access at Springerlink.com

\begin{abstract}
Graphene nanoplatelet (GNP) modified epoxy nanocomposites are becoming attractive to aerospace due to possible improvements in their mechanical, electrical and thermal properties at no weight cost. The process of obtaining reliable material systems provides many challenges, especially at larger scale (a volume effect). This paper reports on the main fabrication stages of GNP-based epoxy composites, namely (i) pre-dispersion, (ii) dispersion, and (iii) post-dispersion. Each stage is developed to show the interest and potential it delivers for property enhancement. Chemical modification of GNP is presented; functionalisation by Triton X-100 shows elastic modulus improvements of the epoxy at low particle content $(\leq 3 \%)$. The post-dispersion step as an alignment of GNP into the epoxy by an electrical field is discussed. The electrical conductivity is below the simulated percolation threshold and an improvement of the thermal diffusivity of $220 \%$ when compared to non-oriented GNP epoxy sample is achieved. The work demonstrates how the addition of functionalised graphene platelets to an epoxy resin will allow it to act as electrical and thermal conductor rather than as insulator
\end{abstract}

Keywords Graphene - Nanocomposites - Electrical conductivity Thermal conductivity · Alignment $\cdot$ Functionalisation $\cdot$ Dispersion

Quentin-Arthur Poutrel

quentin-arthur.poutrel@postgrad.manchester.ac.uk

Matthieu Gresil

matthieu.gresil@manchester.ac.uk

1 i-Composites Lab, School of Materials, University of Manchester, Manchester, UK

2 Aerospace Research Institute, University of Manchester, Manchester, UK 


\section{Introduction}

Composite materials are used in many lightweight applications, mainly in transport (aerospace, automotive) and energy (oil, gas \& wind turbine) industries. Nanoparticles can be used to produce composites in two ways: they can replace the traditional fibre reinforcement, or they can be added to the traditional matrix reinforcement system. Carbon nanoparticles - such as graphene, graphene nanoplatelets (GNP) or carbon nanotubes (CNT) - are well known for their outstanding mechanical, thermal, electrical and optical properties. Graphene is a material obtained by isolation of one sheet of graphite. The first characterisation of a single layer graphene was completed by Geim and Nosovelov in 2007 [1]. Using the "scotch tape" method (mechanical exfoliation), they proved theoretical properties of graphene such as graphene's band structure and its linear relation of dispersion. Since this discovery, further research was completed to exploit the exceptional properties of this material $[2,3]$. Graphene is a $2 \mathrm{D}$ material; if we consider only one single layer graphene (SLG), it compounds of $\mathrm{sp}^{2}$ carbon atoms which form a honeycomb structure. By hybridization of its atoms, SLG possesses a very high carrier charge $(\approx$ $250000 \mathrm{~cm}^{2} /$ V.s $)$ and thermal conductivity ( $\approx 3000$ to $5000 \mathrm{~W} / \mathrm{mK}$ ). Due to its morphological form, graphene has a Young's modulus of $1 \mathrm{TPa}$ tensile strength approaching $130 \mathrm{GPa}$ tensile strength with a density of $0.77 \mathrm{mg} / \mathrm{m}^{2}$ [4]. However, it is extremely difficult to produce inexpensive graphene monolayers without defects, so derived forms of graphene are used with lower properties, such as GNP. GNP are generally composed of ten to a hundred layers of graphene, which leads to lower properties than SLG, but retains some of these including high electron mobility, and high mechanical and thermal performance. The platelet shape of GNP results in edges that are more readily susceptible to chemical modification for an enhanced dispersion, and it provides lower thermal contact resistance, achieving a higher thermal conductivity when included in a polymer matrix. In terms of mechanical behaviour, GNP exhibit good tensile modulus of the order of $1 \mathrm{TPa}$, but possess a lower bending resistance [5].

\subsection{Fabrication Challenges}

The main challenge in manufacturing reproducible nanocomposites is achieving a good control at the nanoscale. To use nanocomposites in real applications, the network of nanoparticles should be perfectly controlled during manufacture, so it is important to control the dispersion and interaction between the reinforcement and matrix. There are many parameters during manufacture that can affect the final properties of the nanocomposite. These include the choice of dispersion technique, processing time, chosen curing cycle, type of nanoparticles, ratio of nanoparticles to matrix, etc. [6-8]. The effects of changing each of these parameters, and more, have been investigated over the last few years and are still not well understood [3, 9]. During manufacturing, it is possible to separate the process in three main categories: (i) predispersion, (ii) mechanical dispersion, (iii) and post-dispersion stage.

In the pre-dispersion manufacturing step, two sub-stages can be identified: (a) simulation, and (b) functionalisation of nanoparticles. Using simulation in physics allows the number of experiments to be reduced and thus time and cost for development at laboratory and industry scale by linking theory and experimentation [10-16]. Functionalisation of nanoparticles, when they are dispersed in an epoxy resin, could have two aims: improving the dispersion quality by decreasing the interaction between nanoparticles [17-19], and improving the mechanical properties of the composite by improving the interfacial bonding between matrix, nanoparticles and micro-reinforcement [20-23]. When nanoparticles are dispersed in an epoxy resin, 
they have a tendency to re-agglomerate and create only weak bonds with the epoxy molecules by Van der Waals interaction. When the composite is loaded, stronger bonds are required at the interface (such as hydrogen bonds) for efficient load transfer between the epoxy and nanoreinforcements [24]. Hydrogen bonds are formed between carboxyl groups in the epoxy molecule and amino groups in the hardener molecule during polymerisation to form a strong tri-dimensional network. Many studies have focused on using these chemical groups or their derivates for the functionalisation of GNP to form stronger interfacial bonds. However, the electrons transport is known to appear mostly on the surface of GNP or CNT [25], so the surface functionalisation can decreases their electrical properties. The choice of functionalisation will depend of the required properties of the composites and its application.

The dispersion step is a crucial fabrication phase for nanocomposites since the tendency of GNP to stack together and form agglomerate reduces their properties and thus the final product $[7,26]$. The mechanical dispersion of CNT has been widely studied $[27,28]$ and can be extend to GNP due to their similar composition and structure.

The post-dispersion step involves control of the nano-network formation before or during the curing process. The alignment of nanoparticles can change and enhance properties of the nanocomposite and obtain preferential orientation properties. Nanoparticles within a polymer often have a random orientation, which can cause random or anisotropic mechanical, electrical, and thermal conductivity of the nanocomposite. Many applications of nanocomposites require preferential orientation such as fracture toughness decreased [29] or deicing property in aircraft [30]. To achieve this, it is important to easily process and control the nano-network formation to manufacture composites with one or several preferential orientation ( $\mathrm{s}$ ) controlled by a relatively simple process. Some studies have been conducted on the orientation of nanoparticles in a matrix using different techniques such as infiltration [31], shear force [32], mechanical stretching [33], electrospinning [34], in-situ polymerisation, or by use of a magnetic or electric field [35].

Figure 1 shows the different steps of the manufacture of GNP/epoxy composites. Many parameters are involve in the manufacture of advanced multifunctional composites based on GNP/epoxy resin; The simulation requires higher development to link theory, to reduces time and cost production. Reducing tries and time for development would allow researcher and industry to understand and develop large scale nanocomposite such as wing or fuselage for aircraft. Predict failure of nanocomposites and proof this failure by simulation and testing will lead to remove the fear of composites in aerospace industry. Define a good functionalisation depending of the product requirement will lead to a wide range of application, from hard joint to high flexible composites with lower strength [36].

The dispersion parameters should be chosen based on the agglomeration tendency and the level of dispersion/damages tolerated for the final nanocomposites. This, therefore, is highly dependent on the initial properties of nanoparticles and matrix, and the interaction between them (e.g. resin viscosity, induced temperature, size and shape of nanoparticles, functionalisation, etc.). The post dispersion treatment of these composites can bring high isotropy and improvement of several properties such as better electrical conductivity [37], reduced fracture toughness for fibre composites [38], but is also dependant on the previous manufacturing steps used. In addition to the mentioned parameters, there are several such as temperature and nanoparticle quality whose effect on the properties of nanocomposites has not been fully investigated. This non-exhaustive list shows that each step of the manufacturing process influences on the following step and final composites properties. To obtain a nanocomposites with high specific properties, each parameter has to be perfectly controlled. 


\section{Predispersion steps}

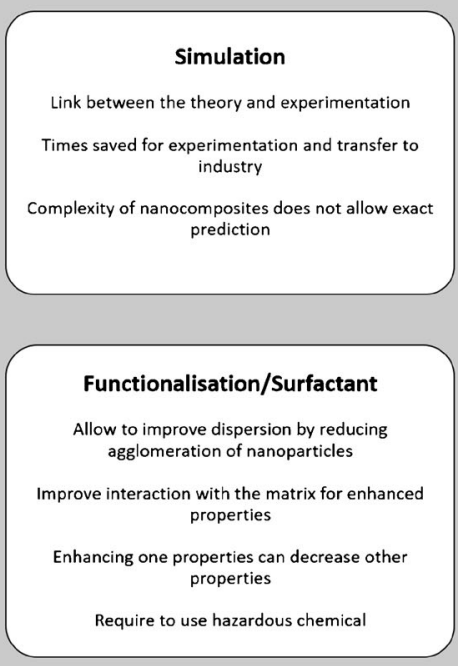

\section{Post dispersion}

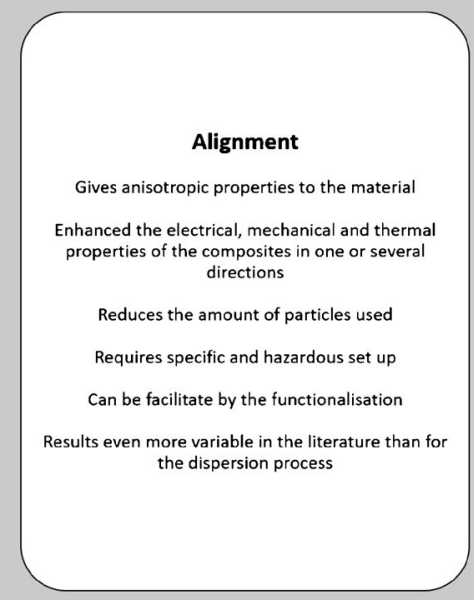

\section{Mechanical dispersion} methods

Calendar process
$\begin{gathered}\text { Good reduction of agglomerates and possible } \\ \text { preorientation of particles } \\ \text { Long process }\end{gathered}$

Limitation of agglomerate size due to the gap size and no homogeneous system

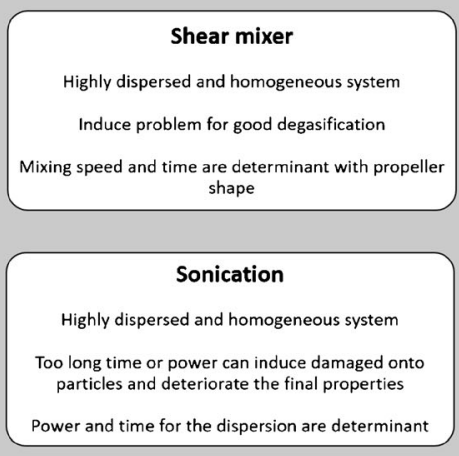

Parameters undeveloped and playing a role all over the manufacture process

\section{Particles:}

Initial properties, shape and size

\section{Matrix: epoxy resin}

Initial properties, viscosity

\section{Epoxy/GNP mix:}

viscosity, temperature, time of addition of hardener

\section{Laboratory conditions:}

Storage condition, type of instrument used for dispersion, room temperature, etc.

Fig. 1 A non-exhaustive list of stages for the fabrication of GNP/epoxy nanocomposites 
This paper presents a review of the three steps of manufacturing graphene/epoxy nano-composites. The possible pre-treatments of nanoparticles before dispersion are introduced, and their influence on the final nanocomposite properties discussed. Then, the main dispersion techniques and their parameters are described. To finish on the possibilities to align the nanoparticles after the dispersion (as post-dispersion step) to obtain preferential direction properties. The mechanical properties of functionalised GNP/epoxy composites show improvement of the interfacial bond, and alignment of GNP using an electrical field show an improvement in thermal diffusivity of $220 \%$ compared to non-oriented GNP/epoxy nanocomposites. Raman spectroscopy and scanning electron microscopy (SEM) are used to confirm the formation of chain structure. The influence of each parameter, and its influence on the final properties of the composites are discussed. This work confirms that the number of parameters and processes available to manufacture advanced multifunctional graphene/epoxy nanocomposites presents many challenges as well as future applications.

\section{Manufacture of Nano-Modified Epoxy Resin}

\subsection{Chemical Modification of Graphene Nanoparticles Before Dispersion into the Epoxy}

A potential way of improving the properties of graphene/epoxy nanocomposites is to add a chemical group on the surface or the edge of the GNP. Graphene has a poor chemical interaction with epoxy resin, and has the tendency to stack together with other graphene particles by Van der Waals interactions; this reduces the quality of the dispersion and thus the final composite properties. The addition of a chemical group onto the particles is called functionalisation. However, it is important to distinguish partial functionalisation (edge functionalisation) with initial aim of improving interfacial bonding between matrix and GNP. And addition of surfactant which is a total surface functionalisation of GNP in a solvent to initially improve the dispersion quality.

\subsubsection{Surfactant}

Surfactants are molecules consisting of hydrophobic and hydrophilic groups. They reduce the surface tension and improve compatibility between nanoparticles and solvent. Depending of the nature of surfactant used, it may act by steric repulsion force, coulombic attraction, physical adsorption or hydrogen bonding [39, 40]. Two categories of surfactant exist: (i) ionic e.g. sodium dodecyl sulphate (SDS) [18], sodium dodecyl benzenesulfonate (NaDDBS) [41, 42]; and (ii) non-ionic e.g. Triton X-100 [17, 19].

Addition of a surfactant increases the solubility of the graphene in a solvent as well as the compatibility between the epoxy resin and nanoparticles [40]. The physical adsorption of the chemical group induces less damages onto the particles than a classic functionalisation which makes this chemical modification really attractive for graphene/ epoxy nanocomposites [24]. Only a few studies have been conducted on the treatment of GNP with a surfactant $[17,18,42]$. However, due to the close structure between CNT and GNP, previous studies made on CNT can be used as a base and inspiration for the functionalisation of GNP. Some studies were made on the advantages of using one kind of surfactant compare to the other [40,42]. Both categories show similar results, but ionic surfactant requires determination of the zero point of charge of the particles and to modify their surface charges $[19,41]$. The chemical structure of the surfactant, such as 
the polarity of the head group or the length of the alkyl chain, determines the influence on the dispersion quality [42]. Bai et al. [43] found that the shorter the hydrophilic segment of surfactant Triton X, the higher the suspendability of the nanoparticles, with the best adsorption quality obtained with Triton-114. Richa et al. [40] studied four different surfactants: Triton X-100, Tween 20, Tween 80, and SDS. Triton X-100 showed the highest dispersion power over other surfactants due to its benzene ring, which enhances the adsorption on nanoparticles by the $\pi-\pi$ interaction. Another study by Islam et al. [42] indicated that NaDDBS has better dispersion ability than Triton X-100. The head group of the NaDDBS, smaller than the head group of the Triton-X, introduces more charge repulsion and thus a better dispersion. Moreover, Gong et al. [44] found that the addition of surfactant induced higher glass temperature transition, and elastic modulus by improving the interfacial bonding between the nano-reinforcements and the epoxy resin. The improvement of the electrical and mechanical properties of the nanocomposites by Triton X-100 was also demonstrated by Geng et al. [20].

\subsubsection{Functionalisation}

To improve the mechanical properties of the nanocomposites, it is necessary to improve the quality of the nanoparticle/epoxy interface. The most common and logical way is to bond the surface of GNP with a functional group which matches the matrix [45]. Prolongo et al. [46] revealed the importance of surface functionalisation by studying the effects on nonfunctionalised GNP on the properties of GNP/epoxy nanocomposites. They showed that, compared to neat epoxy, composites have a constant tensile modulus, and lower tensile strength. The poor surface interaction between the particles and the matrix hinders the load transfer from the epoxy to the reinforcements. Therefore, the nanoparticles act as stress concentrators and deteriorate the mechanical properties of the epoxy. Despite the inert surface of graphene, Sharma et al. [47] showed that some chemical groups can be reactive to it. The effect of bromine functionalised GNP on the mechanical and electrical properties of composites was also studied [22]. The presence of bromine caused the formation of ionic and covalent bonds with the matrix, improving the flexibility of the composites, and the electrical conductivity. However, some studies [25, 48-50] have shown that the surface functionalisation of GNP can also decrease the electrical properties of the nanocomposites compared to when unmodified GNP is used. As the electron displacement is known to occur mainly on the surface of graphene, highly functionalising the surface might increase the mechanical properties but decrease the electrical properties of the final product. The functionalisation of nanoparticles is an important pre-step in the manufacture of nanocomposites. It influences the final properties of nanocomposites. Several studies $[27,45,51-55]$ show modification in the mechanical or electrical properties, and thus functionalised GNP can be used for a wide range of application, depending of the specific requirements such as high tensile stress, flexural strength or high electrical conductivity.

\subsection{Dispersion Methods}

The dispersion of nanoparticles is the major step in the manufacture of graphene based nanocomposites [6]. Due to their small dimension, nanoparticles possess a high surface interaction and have a tendency to stack together and form agglomerates, decreasing the properties of the nanocomposites. It is then necessary to use some dispersion techniques to reduce the agglomerates and obtain a homogeneous solution for stable properties of the 
nanocomposites. Three main techniques are used to disperse GNP into epoxy: (i) sonication; (ii) shear mixing; and (iii) three roll mill or calendar process.

Sonication uses the compression and decompression energy induced by ultrasound to break the Van der Waals interactions between graphene nanoplatelets. This leads to a reduction in the size of agglomerates and particles. However, sonication can introduce some defects into the nanoparticles, which influence the final nanocomposites properties, and it cannot be used for much longer than $30 \mathrm{~min}$ [8]. The variable parameters of this technique are the shape of the probe, the amplitude (power), the time, and the temperature.

Shear mixing uses the shear forces induced by rotation of a propeller to break the agglomerates and obtain a homogeneous solution despite inducing difficulties for the degassing of the resin $[6,56]$. The variable parameters of this technique are the time, the propeller rotation speed, propeller geometry, and temperature.

The calendar process also uses shear force mixing. The first and last rolls rotate in the same direction, and the middle roll rotates in the inverse direction. The gaps between rolls are adjustable which allows regulation of the final dispersion of composite. However, the size of GNP would be too small in relation to the minimum gap between two rolls. This fact leads to not prevent a total agglomeration of GNPs and orientate slightly the larger agglomerate [57].

Often, a combination of two dispersion techniques has been used to obtain the best dispersion quality $[27,28,57,58]$, however in some cases it is possible to obtain the same result with only one technique of dispersion. [59]. Meanwhile, some results in the literature showed variable techniques and results which are dependent of other parameters such as the resin viscosity, initial size, shape and properties of the nanoparticles [60]. Dispersion of nanoparticles by a mechanical technique see presents many challenges for obtaining reliable results; it is necessary to overcome these challenges before attempting to reproduce the processes on an industrial scale $[3,9]$.

\section{Post-Dispersion: Alignment of Nanoparticles}

To obtain the required properties, the GNP/epoxy mixture has to be homogeneous and well dispersed before the curing process. However, some applications benefit from alignment of the nanoparticles to improve preferential orientation properties. Firstly, giving a preferential orientation to the nanoparticles leads to anisotropic electrical and thermal conductivity; some studies enhanced the electrical conductivity by 10 times or more in one direction, compared to the other two [37,61]. This orientation reduces the percolation threshold required for a nanocomposite to be conductive. Increasing the length alignment in one direction reduces the number of particles needed to create a path from one side of the composite to the other. This allows a lower loading of nanoparticles to be used, thus decreasing the composite cost and likelihood of re-agglomeration. In this paper, two techniques of alignment are discussed: (i) electric field [25, 26, 37, 61]; and (ii) a magnetic field [62-69] alignment.

\subsection{Alignment by Electric Field}

Martin et al. [70] studied the orientation of CNT in an epoxy resin under an electric field. They noticed that inside an epoxy system, the surface of CNT is negatively charged, due to the basic 
character of the epoxy system. This charge induces an electrophoretic displacement under a DC electric field. They found that the alignment was better under an AC field due to the dielectrophoresis induced by the non-constant electric field and the Coulomb interaction between the tips of the CNT. This interaction is due to the higher polarisation of the CNT in the axis direction than the radial direction. Further studies have explored the influence of an electric field on the alignment of CNT [25, 37, 61]. Larijani et al. [61] showed that in a polycarbonate matrix the DC field only prevents the aggregation of CNT meanwhile the AC or a magnetic field allows a well alignment. This alignment allows an anisotropic electrical and thermal conductivity as well as improvement of tensile properties due to the load transfer between the matrix and the nano-reinforcements. Wu et al. [37] studied the alignment of GNP under an electric field and showed that the same behaviour could be observed as for CNT. Under an AC field, the anisotropic shape of GNP induced their rotation along the direction of the electric field, then the dipole interaction created by the non-constant field leads to an "endto-end chain" formation. With a controlled network they improved the electrical conductivity by $7-8$ orders of magnitude, the thermal conductivity up to nearly $60 \%$ and $900 \%$ for the fracture toughness, compared to the neat epoxy [37]. To improve the alignment of particles by applying an electric field, Prolongo et al. [25] studied the effect of surface functionalisation by positive and negative charges. They found that both functionalisation led to a quicker and better alignment with modified surface charges. However, when the CNT is wrapped by the surfactant, the electrical properties of the composites decrease compared to a tip functionalisation of the CNTs.

The GNP alignment theory was developed by Wu et al. [37]. When the GNP are dispersed in a dielectric matrix (i.e. epoxy resin), and subjected to a sinusoidal alternating electric field, the GNP are polarised and gain a dipole moment due to the different dielectric properties of the GNP and the epoxy resin. The induced polarisation and the electric field then interact together as a torque (considering the GNP as oblate spheroids with a major radius $a$, and a minor radius $b$, the shape anisotropy inducing a higher polarisation moment along $a$ than the $b$ axis) [37]:

$$
T^{e}=\vec{E} \times \vec{\mu}=E_{\perp} \times \mu-E \times \mu_{\perp}
$$

Where $E_{\|}=E \times \sin \theta$ and $E_{\perp}=E \times \cos \theta$ on the GNP and induce their rotation along the electric field direction. The symbol $\|$ and $\perp$ refer respectively to the graphene plan direction and out of plane. They also developed a model which determines that the time rotation is dependent on the frequency of the applied electric field, the initial angle $\theta_{0}$ between the GNP major axis and the electric field and the drag force applied by the resin (which is dependent of the resin viscosity). After the orientation the GNP are submitted to a new torque [37]:

$$
T^{e}=q \times 2 a \sin \theta
$$

Where $q$ is the electric charge present at the ends of the platelet. This torque tends to attract two edges of GNP with different charges (positives and negatives) and leads to an "end-to-end connection". The time, and the speed of the chain formation is dependent of the applied field frequency, the resin viscosity, and the number of charges on the GNP.

\subsection{Alignment by a Magnetic Field}

Several researcher explored the orientation of CNTs or GNPs under a magnetic field $[37,62-69,71,72]$. When a very strong magnetic field $(\approx 7-10 \mathrm{~T})$ is applied, the 
nanoparticles have the tendency to align themselves along the direction of the magnetic field. The reason of this alignment is the difference of the parallel $\left(\chi_{\|}\right)$ and perpendicular $\left(\chi_{\perp}\right)$ diamagnetic susceptibilities of the CNTs [73]. As $\left|\chi_{\perp}\right|>\left|\chi_{\|}\right|$, the CNTs have a tendency to align with the magnetic field if the applied magnetic force is strong enough to overcome the thermal energy and the Brownian motion induced by the thermal effect. For GNP particles, as $\left|\chi_{\perp}\right|$ (out of plane) is either superior to $\left|\chi_{\|}\right|$(in plane) [74] the same phenomenon should be observed. In the case of a magnetic field or an electrical field, different parameters play a role in the alignment and displacement of the particles in a liquid. The main parameters are the interactions between the particles, the field (magnetic or electric), the viscosity of the resin, the time of the process, and the inter-particles interaction. To improve and facilitate the orientation of nanoparticles, it is necessary to minimise the nanoparticles contents and interaction between them. The field required to align them can present challenges; using a high voltage field requires a lot of energy, complex setup, and specific health and safety precautions. Using a less viscous matrix allows to decrease the field required to align the reinforcements [66] but would not be suitable for aerospace applications. To obtain this requirement, it is possible to use a low viscosity resin or preheat the resin before, with or without the addition of the hardener. When the hardener is added to the resin, the temperature should be high enough to reduce the viscosity without starting the curing process. The major axis of research to decrease the field applied, is to functionalise the CNT or GNP with specific charges or magnetic particles. In the case of a magnetic field, a magnetic (nano) particles has to be bonded/functionalised on the nano-reinforcement to increase the interaction with the field. Several techniques have been developed to functionalise the nanoparticles, for example, using magnetite as magnetic material [67-69], which has some advantages as low cost, easy fabrication process, and avoid more oxidation.

\section{Experimental Methods}

\subsection{Dispersion of Nanoparticles}

Grade $\mathrm{M} 25 \mathrm{xGnP} \circledast$ graphene nano-platelets were purchased from XG Sciences, consisting of short stacks of graphene sheets in a planar form. The average thickness of 18 layers of graphene is $6-8 \mathrm{~nm}$, and a typical surface area of 120 to $150 \mathrm{~m}^{2}$ with average diameters of $25 \mu \mathrm{m}$. The epoxy resin (Araldite LY564) and hardener (Aradur 2954) were provided by Huntsman. Triton X-100 ((C2H4O $\left.)_{n} \mathrm{C} 14 \mathrm{H} 22 \mathrm{O}\right)$ was purchased by Sigma Aldrich. Surfactant was added onto the GNP by dissolving the Triton X-100 in the acetone and sonicated with the GNP for $30 \mathrm{~min}$. The epoxy was then added and the mixture was mixed at $3000 \mathrm{rpm}$ for $2 \mathrm{~h}$. Then, the hardener was added to the mixture, degassed in a vacuum woven and poor in a mould and cured.

\subsection{Alignment of Nanoparticles}

For the alignment, an electric field was applied using a function generator Tektronix AFG 3052 to apply a wide range of frequency and a power range from 0.01 up to $10 \mathrm{Vpp}$. The voltage generator 
was coupled with one amplifier to allow a higher voltage peak of up to $1200 \mathrm{~V}$ to be applied. The preparation of sample for alignment was almost the same except that the GNP was sonicated in the epoxy for $30 \mathrm{~min}$ without acetone or Triton X-100.

\subsection{Mechanical Testing}

Differential Scanning Calorimetry (DSC) was made with Q100 DSC (TA Instruments, USA) to measure modification of glass transition temperature of GNP/epoxy composites. The temperature was increased from $20^{\circ} \mathrm{C}$ to $200^{\circ} \mathrm{C}$ twice at $10^{\circ} \mathrm{C} /$ minute. Tensile properties were measured using Instron Machine. Samples were tested at a crosshead speed of $10 \mathrm{~mm} / \mathrm{min}$ at room temperature. Seven samples were tested for each formulation.

\subsection{Measurement of Electrical and Thermal Properties}

The electrical characterisation was made by two point probe method with a Multimeter Keithley 2000. Infrared (IR) thermography was used to measure the thermal diffusivity $(\alpha)$ of the sample. An external heat source (two flash lamps, delivering a combined pulse of approximately $6 \mathrm{~kJ}$ over a duration of $10.6 \mathrm{~ms}$ ) heats the front face of the sample while an IR camera (Thermosensorik GmbH, Germany) records the thermal radiation (temperature) of the rear surface of each pixel.

\section{Surfactant: Dispersion and Mechanical Results}

\subsection{Reference Samples: raw GNP/Epoxy}

The Young modulus and ultimate tensile strength (UTS) of un-treated GNP/epoxy nanocomposites with different loading of GNP is shown in Fig. 2a and b. Following the expectation, the modulus increases linearly with the amount of nanoparticles until $3 \mathrm{wt} \%$ but decreases at 5 $w t \%$. This decrease might be due to the agglomeration of nanoparticles acting as stress concentrator. Moreover, the viscosity of the resin increases with the amount of particles which decreases the quality of the dispersion/homogenisation of the nanocomposites. In contrast, the UTS decreases with the GNP content (70.44 MPa for pure epoxy samples compared to $37.48 \mathrm{MPa}$ for epoxy with $5 \mathrm{wt} \% \mathrm{GNP}$ ). This confirms the poor interfacial bonding between the epoxy and nanoparticles. These results are similar to Wang et al. [75] and King et al. [76]
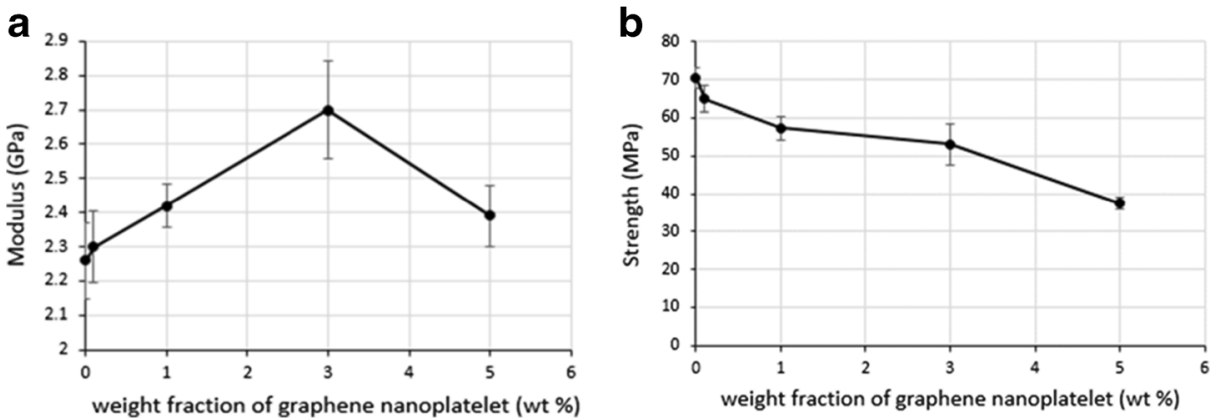

Fig. 2 a Modulus and b strength of GNP based epoxy nanocomposites for different loading of GNP (wt. \%) 
although the value was slightly higher than is found in this paper. This may be due to the curing process, the dispersion method, or the specific type of GNP used, confirming that every single modification in the manufacture of GNP based epoxy nanocomposites have an impact of the mechanical properties.

\subsection{Effect of Surfactant on the Dispersion Quality}

The structure of Triton X-100 is comprises a benzene ring, hydrophilic segment and hydrophobic segment as described in Fig. 3. The hydrophilic segment interacts with epoxy. Benzene rings and hydrophobic groups tend to interact with GNP, as described by Geng et al. for CNT [20]. The hydroxyl, ether and epoxy groups within the epoxy molecule have strong polarity and surfactant have a hydroxyl group, which facilitate the formation of hydrogen bonding between epoxy and surfactant. Benzene ring in surfactant can adsorb on the surface of GNPs because of the $\pi-\pi$ interaction between them. Triton X-100 may act as a bridge between two main composite components so that it is harder for GNP to stack with each other.

GNPs can be wrapped by the surfactant molecules. When the concentration of surfactant is high, GNP and surfactant forms micelle. Following some experiment not shown in this paper, the best ratio GNP: Triton X-100 was found to be $1: 15$. The surfactant has to cover the maximum available surface of the GNP to make bonds with the matrix. However, an excess of surfactant leads to the dilution of the epoxy resin and thus decreases the mechanical properties of the nanocomposites.

The effect of surfactant on the dispersion quality can be visually observed in Figs. 4, 5 and 6. Without Triton X-100, the GNP sediments within a day at the bottom of the pot are clearly shown. However, the functionalised GNP does not show any sedimentation after $24 \mathrm{~h}$ due to the steric repulsive force introduced by the surfactant which are stronger than the Van der Waals interaction between GNP particles.

\subsection{DSC Measurements: Impact on Glass Transition Temperature}

The DSC was used to measure the glass transition temperature $\left(\mathrm{T}_{\mathrm{g}}\right)$ of the nanocomposites. The measurements indicate that the modified-GNP/Triton $\mathrm{X}-100$ /epoxy composites have $\mathrm{T}_{\mathrm{g}}$ decreased by almost $40^{\circ} \mathrm{C}\left(106.28^{\circ} \mathrm{C}\right.$ compared to $144.15^{\circ} \mathrm{C}$ for the neat epoxy). As the Triton $\mathrm{X}-100$ molecules are smaller than the epoxy resin molecules, modified GNP (micelles) leave more free volume for the long polymer chains in the resin and increase the likelihood for bonding to them. The polymer chains will bond quicker to other chains or to the Triton X-100,

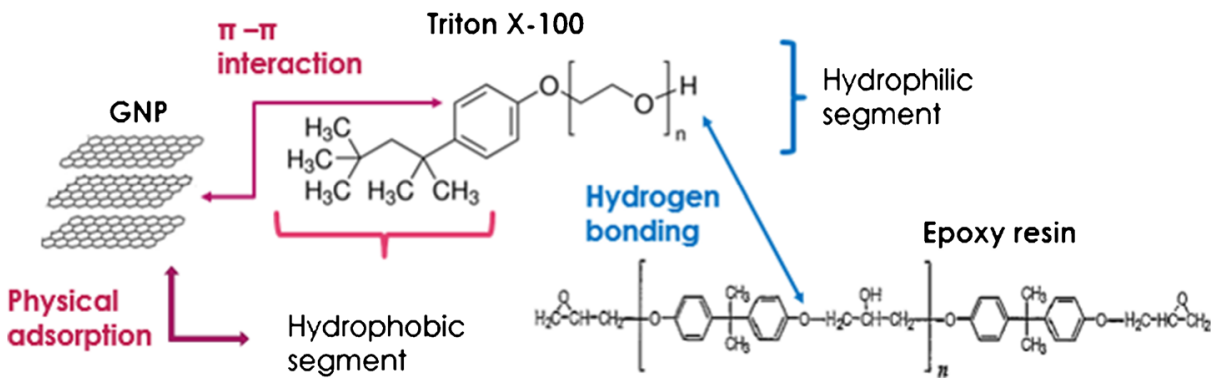

Fig. 3 Schematic of the effect of Triton X-100 molecule in GNP/epoxy nanocomposites 

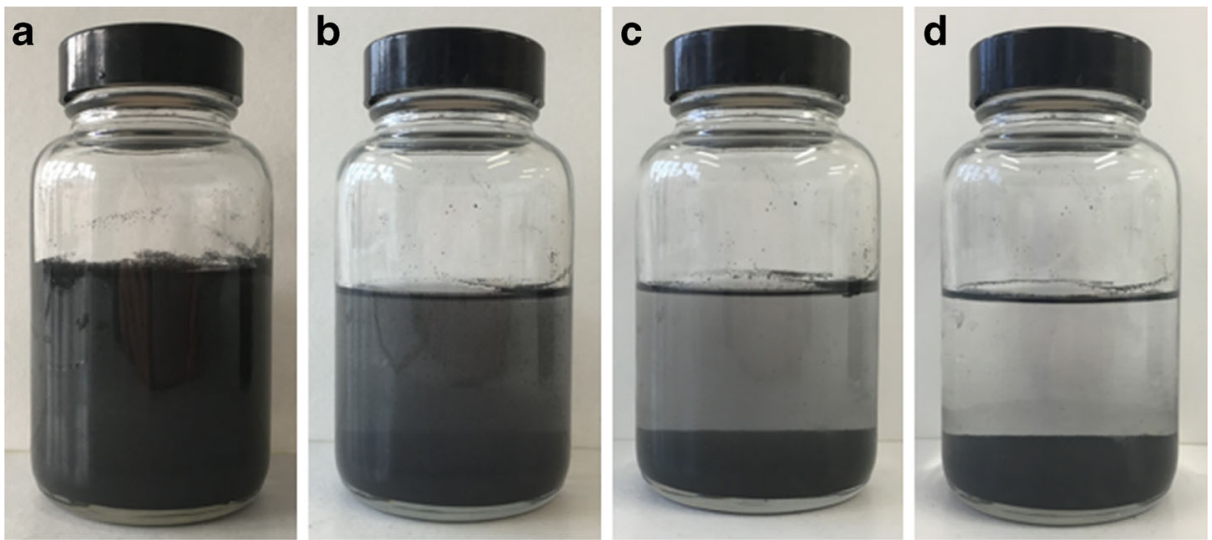

Fig. 4 Dispersion state of GNPs in acetone without the dispersion technique at $\mathbf{a} \mathrm{t}=0 ; \mathbf{b} \mathrm{t}=10 \mathrm{~min} ; \mathbf{c} \mathrm{t}=1 \mathrm{~h}$ and $\mathbf{d}$ $\mathrm{t}=24 \mathrm{~h}$

leading to a decreased $\mathrm{T}_{\mathrm{g}}$. The surfactant effect on $\mathrm{T}_{\mathrm{g}}$ confirms that the epoxy resin is well bonded with the Triton X-100.

\subsection{Triton-/GNP/Epoxy Samples}

The GNP and Triton X 100 were added to the epoxy in a ratio of 1:15 by weight. Figure 7 shows the Young's modulus and ultimate tensile strength (UTS) of the nanocomposites made with functionalised GNP. This results follow the same trend as the un-functionalised GNP nanocomposites. However, the modulus starts decreasing at $3 \mathrm{wt} \%$ instead of $5 \mathrm{wt} \%$. This might be due to: (i) increasing the amount of GNP changes the viscosity of the mixture so the same level of functionalisation on the GNP surface was not achieved using the same parameters; (ii) the volume of modified GNP is larger than before modification, reducing the possibilities of cross-linking during the curing process, which then reduces the mechanical properties of the nanocomposites.

Until $1 \mathrm{wt} \%$, both the stiffness and UTS have been improved by the functionalisation of GNP, suggesting that the interfacial bond between the epoxy and the functionalised GNPs is stronger than that of raw GNP with epoxy. Moreover, due to the higher dispersion quality, no agglomerates are
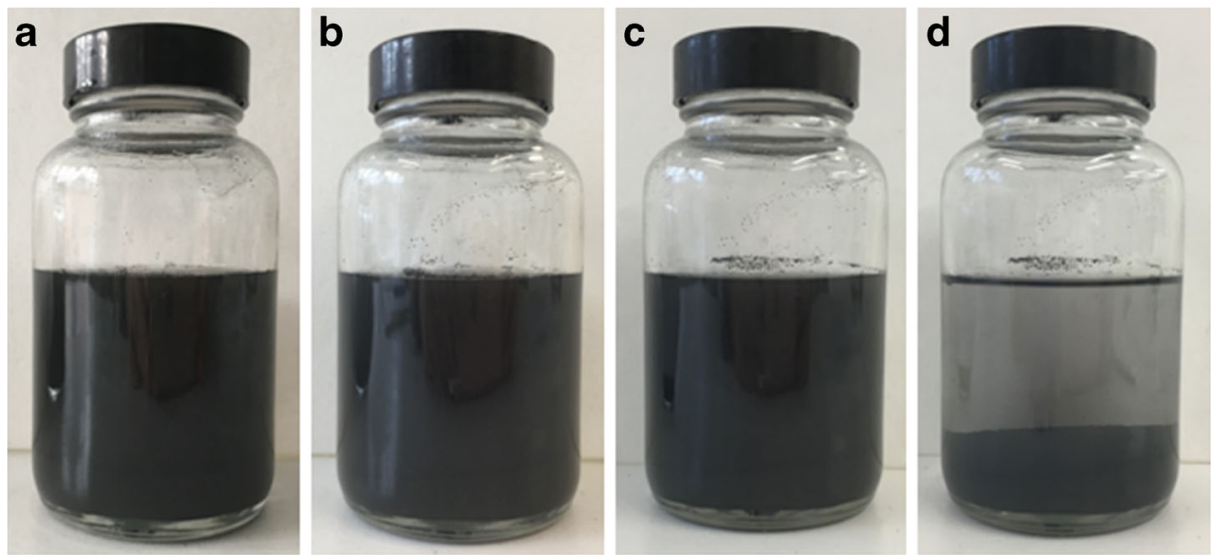

Fig. 5 Dispersion state of GNPs in acetone after sonication for $30 \mathrm{~min}$ at $\mathbf{a} t=0 ; \mathbf{b} t=10 \mathrm{~min}$; $\mathbf{c} \mathrm{t}=1 \mathrm{~h}$ and $\mathbf{d} \mathrm{t}=24 \mathrm{~h}$ 

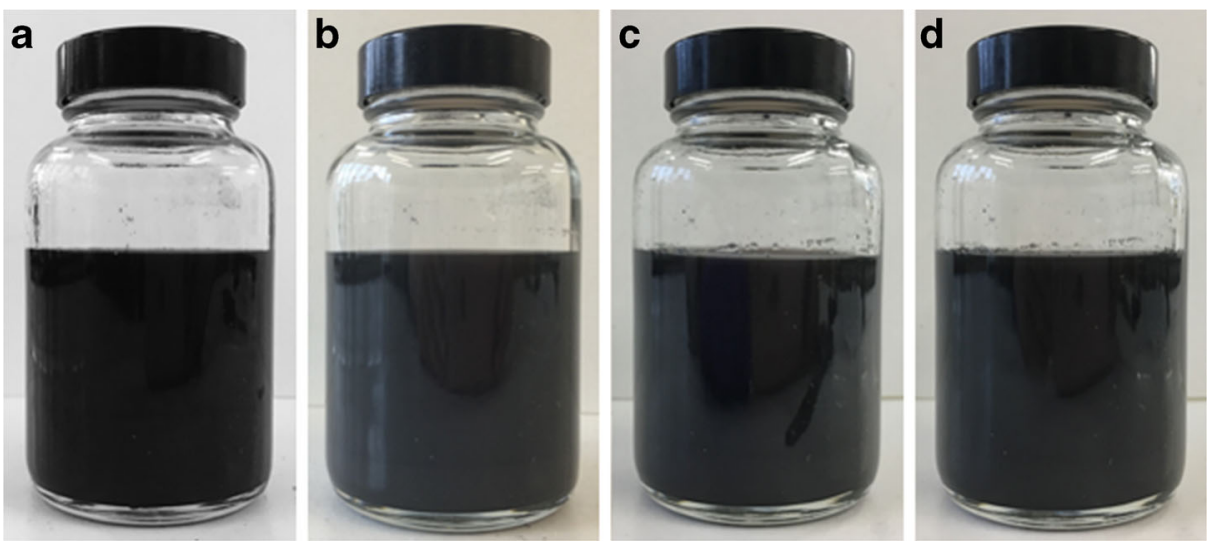

Fig. 6 Dispersion condition of GNPs in acetone with aid of Triton X-100 after sonication for $30 \mathrm{~min}$ at $\mathbf{a} \mathrm{t}=0$; $\mathbf{b}$ $\mathrm{t}=10 \mathrm{~min} ; \mathrm{c} \mathrm{t}=1 \mathrm{~h}$ and $\mathbf{d} \mathrm{t}=24 \mathrm{~h}$

present within the composites. The decrease in mechanical properties at $3 \mathrm{wt} \%$ confirms that complete functionalisation of GNP was not achieved, and that cross-linking of the resin is influenced by the presence of the partially modified GNP. The un-functionalised GNP may act as a stress concentrator which induces defects in the nanocomposites. This conclusion confirms that the manufacture of nanocomposites can be affected by changing one parameter such as the loading of nanoparticles. This modification requires adaptation of the dispersion method to obtain the best composites properties. Figure 8 shows that the dispersion of GNP $(0.5 \mathrm{wt} \%)$ in epoxy with the surfactant is better than that of GNP in $3 \mathrm{wt} \% \mathrm{GNP} /$ epoxy sample containing the surfactant. The higher presence of black area within the $3 \mathrm{wt} \%$ sample confirm that the partially functionalised GNP agglomerated. Their agglomeration left more free volume not accessible to the long polymer chain. These empty volume acts as a stress concentrator which explain the inferior mechanical properties of surfactant-treated $3 \mathrm{wt} \%$ GNP/epoxy sample.

\section{Post-Dispersion Results With Alignment}

It is possible to improve further the properties of nanocomposites by aligning them using an electrical field after dispersion, but before curing. Alignment is done using a frequency of $100 \mathrm{kHz}$ with $1000 \mathrm{Vpp}$ amplitude with a square signal to encourage dipole formation and
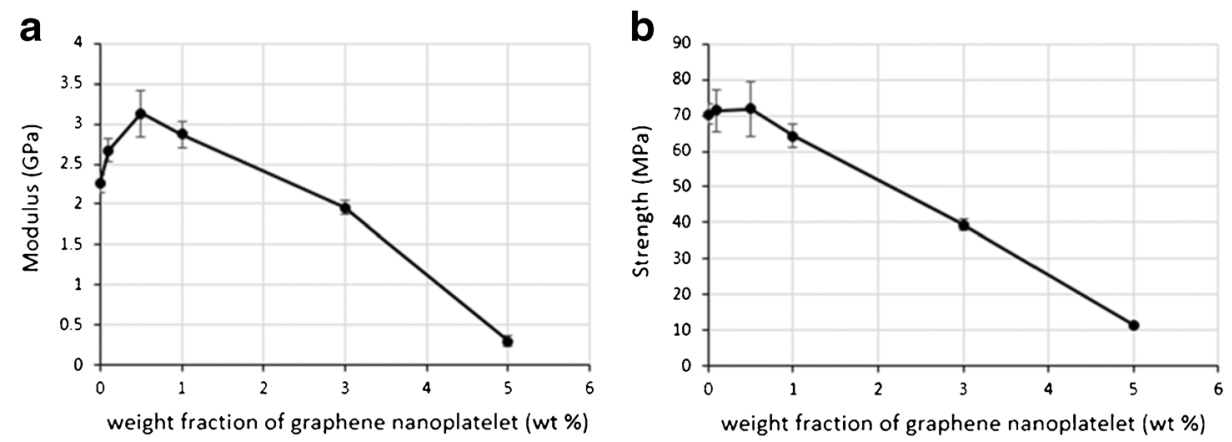

Fig. 7 a Modulus and $\mathbf{b}$ strength of treated-GNP based epoxy composites 


\section{Empty spaces}
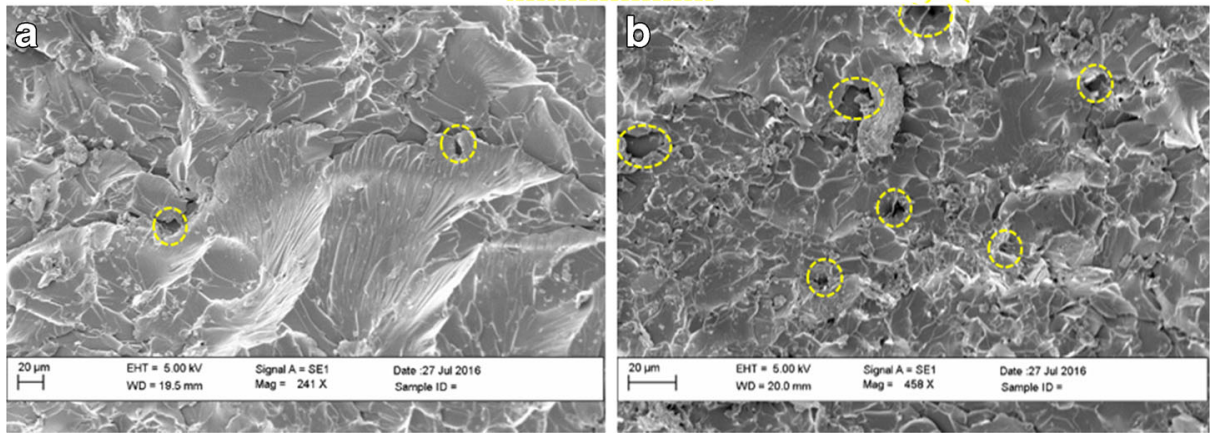

Fig. 8 SEM images of fracture surface of a 0.5 wt $\%$ and $\mathbf{b} 3 w t \%$ of GNP: Triton-100/epoxy

interaction between the GNP particles within the resin. A schematic of the set-up is shown in Fig. 9. This alignment process was applied to samples between 30 to $50 \mathrm{~mm}$.

Several steps were completed before it was possible to form a conductive path of nanoparticles. The minimum required electrical field was found to be around $11 \mathrm{kV} / \mathrm{m}$ depending on the distance between electrodes on the preliminary optical slide experiments. In-situ optical microscopy allowed observation of the movement of GNPs in the liquid epoxy resin at different frequencies.

At $10 \mathrm{~Hz}$, a progressive alignment of GNP is observed as depicted in Fig. 10. The formation of a chain-like graphene network in the direction of the applied electric field is clearly shown. A network of aligned GNP began to develop after approximately $1 \mathrm{~min}$ of exposure to the electric field. A well-defined chain-like structure of GNP extending between the positive and the negative electrodes formed after $7 \mathrm{~min}$. This alignment process is the result of dipole-dipole interaction between the GNPs

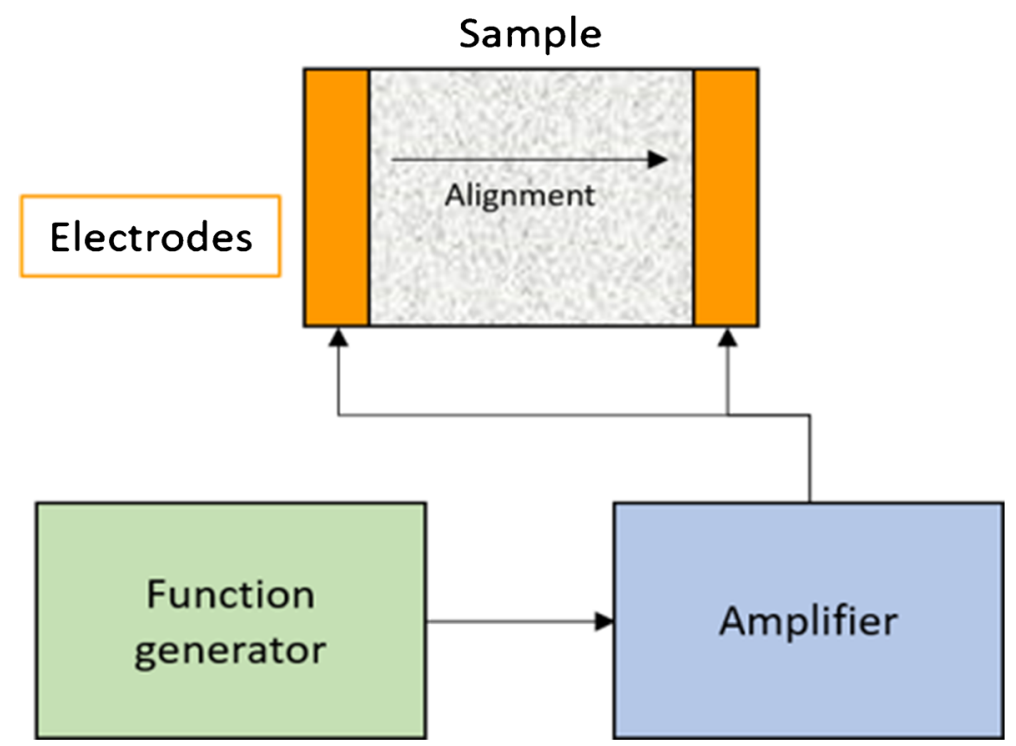

Fig. 9 Schematic of electrical set-up for nanoparticle alignment 


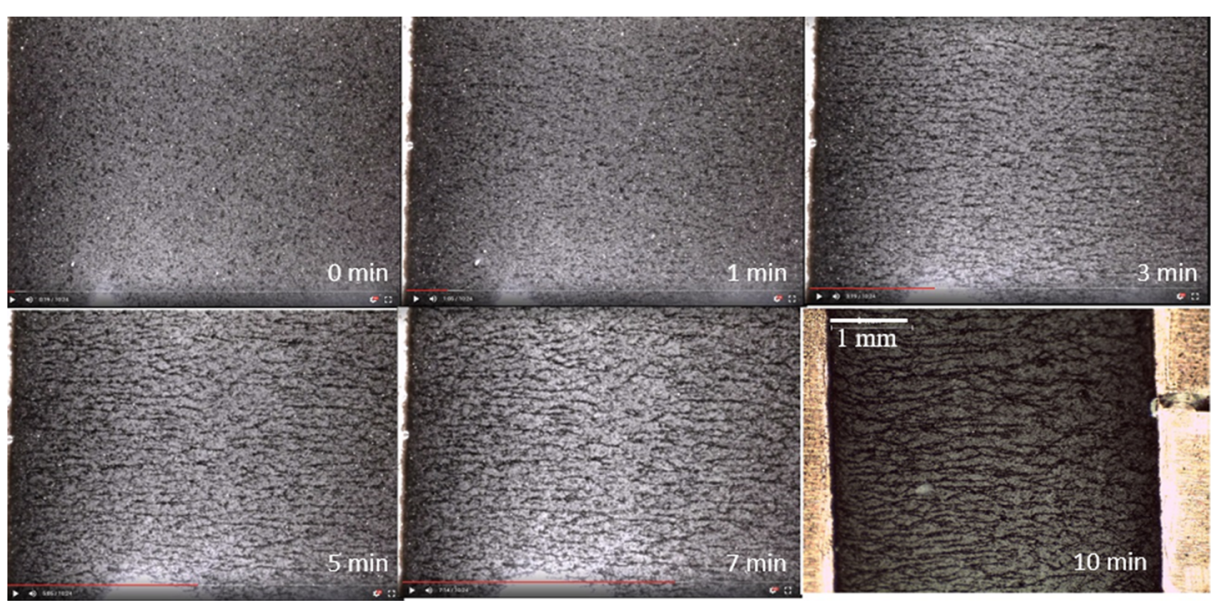

Fig. 10 Optical micrographs of the progression of the alignment of $1 \mathrm{wt} \%$ of GNP in epoxy resin during the application of the square AC electric filed at $10 \mathrm{~Hz}$. Comment: the recorded area is $4 \mathrm{~mm}$

and the electric field. Due to the opposite charges at their ends, they gradually stretch, move closer and connect end-to-end, forming these chain-like structures. These aligned samples were not electrically conductive.

At $100 \mathrm{kHz}$, a different reaction happened and lead to a quasi-instantaneous alignment of nanoparticles as described in Fig. 11. At $11 \mathrm{~min} 39 \mathrm{~s}$, the process of alignment start, then all the GNP in the liquid epoxy started to move very quickly to form at $12 \mathrm{~min} 01 \mathrm{~s}$ thick aligned bundles of GNP. Moreover, all of these samples were electrically conductive. It's likely that the energy provided at $100 \mathrm{kHz}$ was high enough to form end-to-end connections and overcome the repulsion due to the presence of oxygen at the edge of GNP. Once these parameters had been defined it was possible to begin experiments on bulk samples and characterise aligned GNP based epoxy nanocomposites.

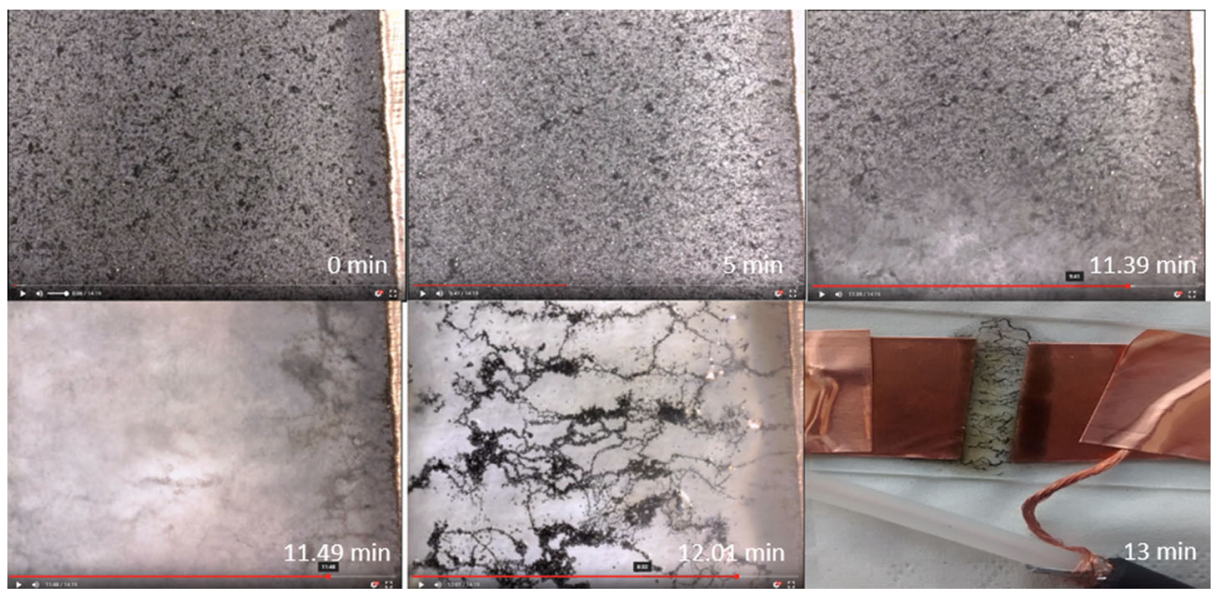

Fig. 11 Optical micrographs of the instantaneous alignment of $1 \mathrm{wt} \%$ of GNP in epoxy resin during the application of the square $\mathrm{AC}$ electric field at $100 \mathrm{kHz}$. Comment: the recorded area is $4 \mathrm{~mm}$ 


\subsection{Characterisation of Alignment Through Fracture Surface Analysis}

The scanning electron micrographs of the fracture surface of the $1 \mathrm{wt} \% \mathrm{GNP} /$ epoxy nanocomposites without the application of an electric field is shown in Fig. 12. As expected, the GNPs are randomly oriented and distributed, with different sizes. The fracture surface follows the geometry of the GNP showing that the interfacial bonding was weak without surfactant treatment, the presence of more black area confirms that the agglomeration was higher without the surfactant. Figure 13 shows the microstructure of 1 wt $\%$ of GNP/epoxy nanocomposites following exposure to the electric field at $100 \mathrm{kHz}$. Most of the GNPs are well aligned, very close to being parallel to the applied electric field direction. Voids and dispersed multi-layered GNPs are also observed. The appearance of the voids could be due to the relatively high stresses generated during the post-dispersion and curing processes. The electric field has aligned the particles and the dipole interaction has enabled the particles to form end-to-end connections, leading to a straight path of GNP.

\subsection{Electrical Conductivity of Nanocomposites}

Table 1 gives the measured values for electrical conductivity on GNP/epoxy composites. Nonaligned raw GNP and Triton X-100 epoxy samples were both electrical insulator, contrary to some values showed in the literature, this fact is probably due to the poor quality of GNP used during these experiments but, also confirms the theoretical result obtained by simulation where the required weight loading was $8.53 \%$ of GNP [77]. These loading of GNP was chosen to have the maximum GNP with a low viscoelasticity. Moreover, the treatment with surfactant, did not improved the electrical conductivity. Aligned samples were all conductive and the percolation threshold was obtained under the simulation values, confirming that the alignment of GNP reduces the amount of particles required. However the result, do not follow the theory, increasing the loading of particles should increase the electrical conductivity of composite and the conductivity in the direction of the applied field should be higher than out of plane. Several parameters might be involved in these results: (i) the higher electrical conductivity out of the applied field direction is potentially due to the high current passing though the sample when the conductivity was obtained. This high current induced damages though the chain structures, and decreased their conductivity while the interconnection out of plane were preserved. (ii) The lowest conductivity with $5 \mathrm{wt} \%$ of nanoparticles is
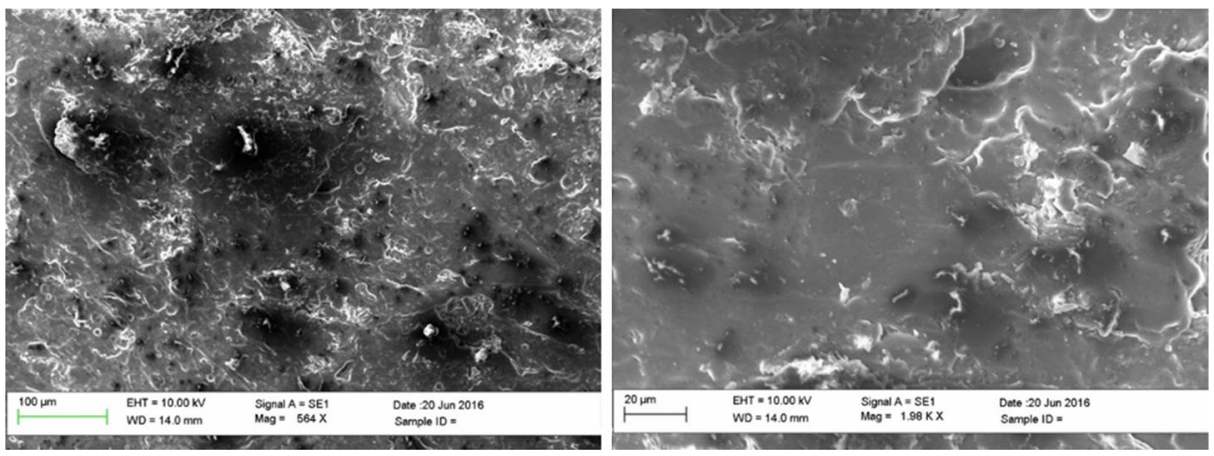

Fig. 12 SEM images of fracture surface of non-aligned GNP based epoxy composite (1 wt\%): GNP show independent direction and no specific connection between each other 

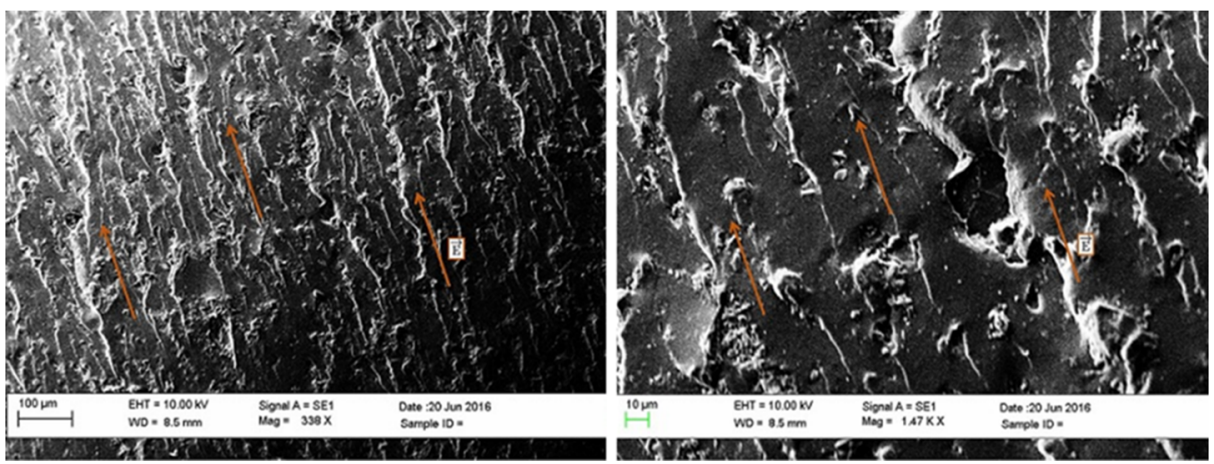

Fig. 13 SEM images of fracture surface of aligned GNP based epoxy composite (1 wt $\%$ ): chain of GNP though the composite along the applied electric field

possibly due to the formation of bigger fillers (due to the dispersion), which have properties closer to graphite than graphene, inducing a reduction of the electrical conductivity though the composites.

To obtain expected electrical conductivity for the final composite, an adaptation of the applied electrical field power and time of application should be used depending of the loading of particles.

\subsection{Effect of Alignment on the Thermal Properties of Composites}

The thermal diffusivity of the composites was measured using active infrared thermography. The thermal properties of a nanocomposite is determined by the phonon propagation, as the phonon scattering is not the same between two particles and between the particles and the matrix (resin), the presence of agglomerates of nanoparticles will lead to the apparition of "hot spot". The phonon scattering is better in the plane of graphene and between two graphene particles than between the particles and the matrix, due to the phonon mismatching [78]. Contrary to the electrical conductivity, the thermal conductivity does not possess a percolation threshold and should increase proportionally with the content of GNPs or nanoparticles [78].

Table 2 shows that the thermal diffusivity is higher in the direction of the applied field, confirming that the GNP were oriented in direction of their plane. As the phonon scattering is higher along the plane of the GNP, the thermal conductivity will be higher when the GNP have their planes oriented in the same direction and connect with each other, acting like an "easy" path for the phonons. The difference is clear for the sample with $5 \mathrm{wt} \%$ of GNP where the electrical field was applied (i.e. aligned). The mean value of the diffusivity is equal to $\alpha=6.5 \times 10^{-7} \mathrm{~m}^{2} / \mathrm{s}$ and $\alpha=2.9 \times 10^{-7} \mathrm{~m}^{2} / \mathrm{s}$ on the panel where the particles were not oriented. This corresponds to an

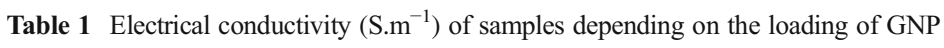

\begin{tabular}{llll}
\hline Direction of measurement & \multicolumn{2}{l}{ Loading of GNP } & \\
\cline { 2 - 4 } & 1 wt.\% & 2 wt.\% & 5 wt.\% \\
\hline Along the applied electric field & $1.05 \times 10^{-6}$ & $3.90 \times 10^{-4}$ & $1.71 \times 10^{-7}$ \\
Out of the applied field direction & $8.05 \times 10^{-5}$ & $5.52 \times 10^{-4}$ & $9.07 \times 10^{-5}$ \\
Non oriented samples & Insulator & Insulator & Insulator \\
\hline
\end{tabular}


Table 2 Thermal diffusivity $\left(\mathrm{m}^{2} / \mathrm{s}\right)$ of samples depending on the loading of particles

\begin{tabular}{llll}
\hline Direction of measurement & \multicolumn{2}{l}{ Loading of GNP } & \\
\cline { 2 - 4 } & $1 \mathrm{wt} \%$ & $2 \mathrm{wt} \%$ & $5 \mathrm{wt} \%$ \\
\hline Along the applied electric field & $3.3 \times 10^{-7}$ & $4.1 \times 10^{-7}$ & $6.5 \times 10^{-7}$ \\
Out of the applied field direction & $2.5 \times 10^{-7}$ & $3.5 \times 10^{-7}$ & - \\
Non oriented samples & $1.9 \times 10^{-7}$ & - & $2.9 \times 10^{-7}$ \\
\hline
\end{tabular}

improvement of $225 \%$ of the thermal conductivity for an oriented panel than a non-oriented samples with 5 wt $\%$ of GNPs.

These experiments show that the post-dispersion step in the manufacture of nanocomposites can have a significant influence on the final properties of the material. Despite a good enhancement of these properties, several parameters such as the type of particles, the temperature, the viscosity of the mixture, and the loading of particle, etc. have an impact on this post-dispersion step and thus on the final properties of the nanocomposites, requiring adjustment in each case to produce reliable composites.

\subsection{Raman Spectroscopy to Characterise the Alignment of GNP/Epoxy}

The Raman spectroscopy was done using a Renishaw InVia Raman microscope, using a spot laser size within 1 to $2 \mu \mathrm{m}$. The three peaks commonly used to characterise the graphene and its derivatives are called the $\mathrm{G}$ peak $\left(\approx 1581 \mathrm{~cm}^{-1}\right)$ associated with the longitudinal vibration of the carbon atoms; the D peak $\left(\approx 1310 \mathrm{~cm}^{-1}\right)$ associated with defects such as functionalisation, edge effect; and $2 \mathrm{D}$ peak $\left(\approx 2680 \mathrm{~cm}^{-1}\right)$ called the defect free peak of graphene. These peaks for the GNPs used in the experiment are shown in Fig. 14a.

The broadening of the $G$ and $D$ peaks indicates the presence of some defects on the graphene nanoplatelets such as oxygen groups. As the oxygen is highly electronegative,, its presence may explain the tendency of the GNP to move from the positive to the negative electrode during the experiment. The presence of the $2 \mathrm{D}$ peak confirms that the platelets
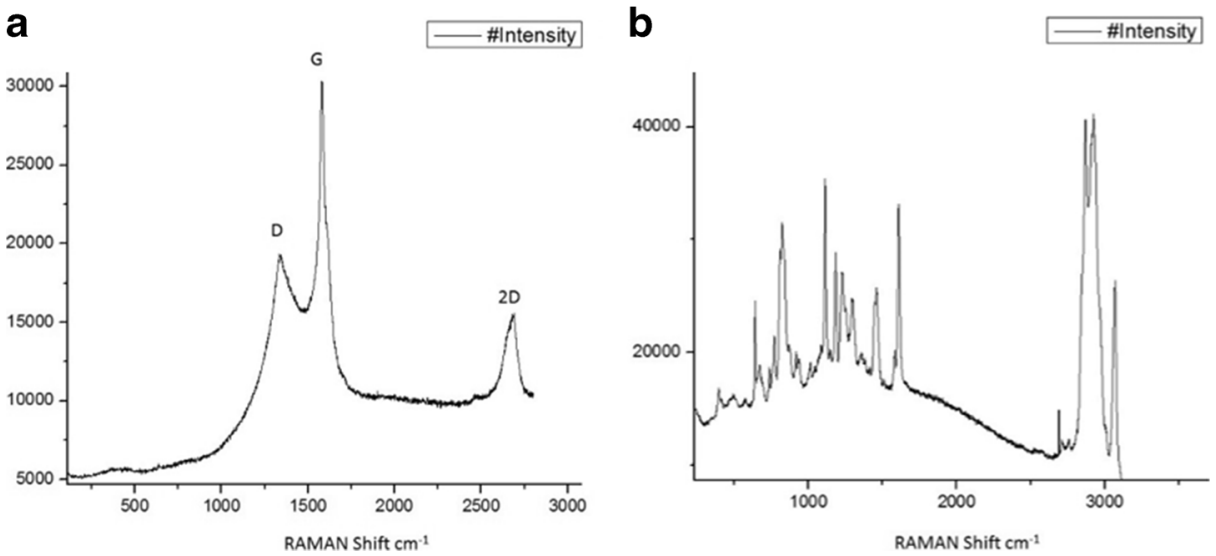

Fig. 14 Raw Raman spectrum: a GNP powder; b neat epoxy 

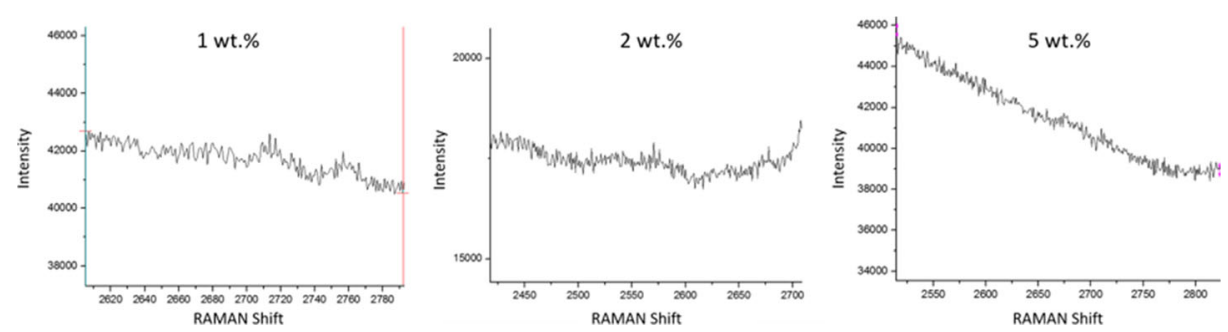

Fig. 15 Absence of the graphene 2D peak in the direction of the applied field for a $1 \mathrm{wt} \%$, b $2 \mathrm{wt} \%$ and $\mathbf{c} 5 \mathrm{wt} \%$ of GNP

consist of 15-20 layers of graphene. In Fig. 14b, the Raman spectrum of the resin is covering almost all the characteristic peaks of the GNP.

After fitting, the peaks of the Raman spectrum of the GNP were defined. It is possible to see that the bi-Gaussian function used for the fitting of the curves was not perfect due to the asymmetry of the peaks and the baseline. From the G and D peaks, even with the fitting, it was almost impossible to obtain some information about the alignment of nanoparticles inside the resin. However, after analysis some information can be deduced from the $2 \mathrm{D}$ peak. This peak is located at $\approx 2665 \mathrm{~cm}^{-1}$ on the Raman spectrum of raw GNP. The $2 \mathrm{D}$ peak $\left(\approx 2665 \mathrm{~cm}^{-1}\right)$ disappeared when the Laser beam was along the GNP chain structures aligned by the applied electric field as shown in Fig. 15.

Figure 16 shows the fitting of the 2D peak for $1 \mathrm{wt} \%$ and $2 \mathrm{wt} \%$ of GNP out of the applied field direction and the fitting for $5 \mathrm{wt} \%$ of non-oriented GNP. When the laser beam is directed out of the applied field direction the 2D peak is present as well as in the non-oriented samples. The absence of 2D peak confirms a chain structure along the incident beam. The GNP aligned and connected in the direction cannot enter in resonance and so the 2D peak is not visible. This peak is specific of low defect graphene; each connection between GNP acts as a defect along the structure, removing the presence of the 2D peak compared to in a non-aligned sample or out of the applied field direction.

\section{Concluding Remarks and Perspectives}

Pre-dispersion, dispersion and post dispersion steps of the manufacture of GNP/epoxy composites have been developed. The choice of possibilities to modify, improve and obtain enhanced properties by the addition of GNP to the epoxy resin are infinite and leaves as limits only imagination and technical process.

The pre-dispersion step allows the initial properties of the nano-reinforcement to be influenced, leading to improved interfacial bonding with the matrix, and thus of the final
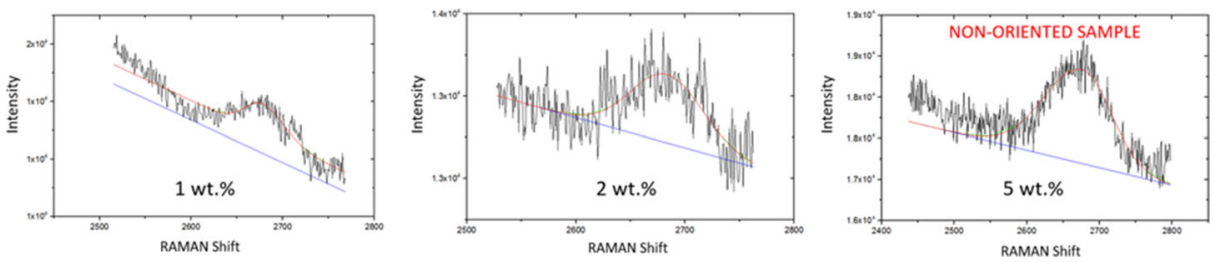

Fig. 16 Presence of the graphene 2D peak out of the direction of the applied field for a) $1 \mathrm{wt} \%$ of GNP, $2 \mathrm{wt} \%$ of GNP and for $5 \mathrm{wt} \%$ of non-oriented GNP 
properties of the composite. However, affecting the initial particle properties could also decrease some properties of the final composite. Each parameter has to be taken into account during the functionalisation, such as the loading of particles included in the final composite. The functionalisation process cannot be the same for a composite at low weight fraction as that for a high weight fraction system. The dispersion is improved by the presence of surfactant by modification of interaction between GNP and DSC with mechanical measurement confirmed improvement of the bond between nano-reinforcement and matrix.

The choice of dispersion method and its parameters remain essential in the manufacture of these composites and play a determinant role in their properties. Even if not fully developed in this study, the parameters of dispersion should be adapted depending on several variables such as the loading of particles, the temperature induced by the dispersion or even the volume of composite prepared.

The post dispersion treatment shows impressive improvements in electrical and thermal properties of the composite (up to $220 \%$ increase in thermal diffusivity at $5 \mathrm{wt} \%$ ), and allows anisotropic properties to be obtained, making these materials suitable for specific applications such as de-icing or lightning strike protection if applied to aircraft skin. Raman spectroscopy and SEM confirmed the formation of "chain" structures along the direction of the applied field. However, this requires use of a strong electrical or magnetic field which can add to the production cost.

Besides the controllable parameters of the manufacture, many other factors can also influence the composite properties such as the purity of nanoparticles, the storage condition of constituents, the temperature and humidity during fabrication, scale (size) effects, in addition to the curing and cooling cycles. These parameters have not been studied within the body of this work, and leave yet again many possibilities and variables in the manufacture of GNP based composites with tailored properties.

Acknowledgments The authors would like to acknowledge financial and technical support from the National Graphene Institute (NGI) and the Aerospace Research Institute (ARI) at the University of Manchester for the completion of this research. Moreover, I would like to acknowledge Hilbert Malada for his support for the understanding of functionalisation.

Open Access This article is distributed under the terms of the Creative Commons Attribution 4.0 International License (http://creativecommons.org/licenses/by/4.0/), which permits unrestricted use, distribution, and reproduction in any medium, provided you give appropriate credit to the original author(s) and the source, provide a link to the Creative Commons license, and indicate if changes were made.

\section{References}

1. Novoselov, K.S., Geim, A.K., Morozov, S.V., Jiang, D., Zhang, Y., Dubonos, S.V., Grigorieva, I.V., Firsov, A.A.: Electric field effect in atomically thin carbon films. Science 80(306), 666-669 (2004). doi:10.1126/science.1102896

2. Signh, V., et al.: Graphene based materials: past, present and future. Prog. Mater. Sci. 56, 1178-1271 (2011)

3. Ferrari, A.C., Bonaccorso, F., Falko, V., Novoselov, K.S., Roche, S., Bøggild, P., Borini, S., Koppens, F., Palermo, V., Pugno, N., Garrido, J.A., Sordan, R., Bianco, A., Ballerini, L., Prato, M., Lidorikis, E., Kivioja, J., Marinelli, C., Ryhänen, T., Morpurgo, A., Coleman, J.N., Nicolosi, V., Colombo, L., Fert, A., GarciaHernandez, M., Bachtold, A., Schneider, G.F., Guinea, F., Dekker, C., Barbone, M., Galiotis, C., Grigorenko, A., Konstantatos, G., Kis, A., Katsnelson, M., Beenakker, C.W.J., Vandersypen, L., Loiseau, A., Morandi, V., Neumaier, D., Treossi, E., Pellegrini, V., Polini, M., Tredicucci, A., Williams, G.M., Hong, B.H., Ahn, 
J.H., Kim, J.M., Zirath, H., van Wees, B.J., van der Zant, H., Occhipinti, L., Di Matteo, A., Kinloch, I.A., Seyller, T., Quesnel, E., Feng, X., Teo, K., Rupesinghe, N., Hakonen, P., Neil, S.R.T., Tannock, Q., Löfwander, T., Kinaret, J.: Science and technology roadmap for graphene, related two-dimensional crystals, and hybrid systems. Nanoscale 7, 4598-4810 (2014). doi:10.1039/C4NR01600A

4. Yue, L., Pircheraghi, G., Monemian, S.A., Manas-Zloczower, I.: Epoxy composites with carbon nanotubes and graphene nanoplatelets - dispersion and synergy effects. Carbon N. Y. 78, 268-278 (2014). doi:10.1016/j. carbon.2014.07.003

5. Lindsay, L., Broido, D.A., Mingo, N.: Diameter dependence of carbon nanotube thermal conductivity and extension to the graphene limit. Phys. Rev. B - Condens. Matter Mater. Phys. 82, 161402 (2010). doi:10.1103/PhysRevB.82.161402

6. Hu, N., Masuda, Z., Yamamoto, G., Fukunaga, H., Hashida, T., Qiu, J.: Effect of fabrication process on electrical properties of polymer/multi-wall carbon nanotube nanocomposites. Compos. Part A Appl. Sci. Manuf. 39, 893-903 (2008). doi:10.1016/j.compositesa.2008.01.002

7. Martin, C.A., Sandler, J.K.W., Shaffer, M.S.P., Schwarz, M.K., Bauhofer, W., Schulte, K., Windle, A.H.: Formation of percolating networks in multi-wall carbon-nanotube-epoxy composites. Compos. Sci. Technol. 64, 2309-2316 (2004). doi:10.1016/j.compscitech.2004.01.025

8. Montazeri, A., Chitsazzadeh, M.: Effect of sonication parameters on the mechanical properties of multiwalled carbon nanotube/epoxy composites. Mater. Des. 56, 500-506 (2014). doi:10.1016/j. matdes.2013.11.013

9. Kostopoulos, V., Masouras, A., Baltopoulos, A., Vavouliotis, A., Sotiriadis, G., Pambaguian, L.: A critical review of nanotechnologies for composite aerospace structures. CEAS Sp. J. (2016). doi:10.1007/s12567-016-0123-7

10. Spanos, K.N., Anifantis, N.K.: Finite element prediction of stress transfer in graphene nanocomposites: the interface effect. Compos. Struct. 154, 269-276 (2016). doi:10.1016/j.compstruct.2016.07.058

11. Parashar, A., Mertiny, P.: Multiscale model to investigate the effect of graphene on the fracture characteristics of graphene/polymer nanocomposites. Nanoscale Res. Lett. 7, 595 (2012). doi:10.1186/1556-276X-7-595

12. Oskouyi, A.B., Mertiny, P.: Monte Carlo model for the study of percolation thresholds in composites filled with circular conductive nano-disks. Procedia Eng (2011). doi:10.1016/j.proeng.2011.04.068

13. G. Ambrosetti, N. Johner, C. Grimaldi, A. Danani, P. Ryser, Percolative properties of hard oblate ellipsoids of revolution with a soft shell, Phys. Rev. E - Stat. Physics, Plasmas, Fluids, Relat. Interdiscip. Top. 78 (2008) 061126:1-061126:11. doi:10.1103/PhysRevE.78.061126

14. Otten, R.H.J., Van Der Schoot, P.: Connectivity percolation of polydisperse anisotropic nanofillers. J. Chem. Phys. (2011). doi:10.1063/1.3559004

15. Vovchenko, L., Vovchenko, V.: Simulation of percolation threshold in composites filled with conducting particles of various morphologies. Materwiss. Werksttech. 42, 70-74 (2011). doi:10.1002/mawe.201100734

16. Hicks, J., Behnam, A., Ural, A.: A computational study of tunneling-percolation electrical transport in graphene- based nanocomposites a computational study of tunneling-percolation electrical transport in graphene-based nanocomposites. Appl. Phys. Lett. 213103, 18-21 (2009). doi:10.1063/1.3267079

17. Wang, H., Wang, H., Zhou, W., Zhou, W., Ho, D.L., Ho, D.L., Winey, K.I., Winey, K.I., Fischer, J.E., Fischer, J.E., Glinka, C.J., Glinka, C.J., Hobbie, E.K., Hobbie, E.K.: Dispersing single-walled carbon nanotubes with surfactants: a small angle neutron scattering study. Nano Lett. 4, 1789-1793 (2004). doi: $10.1021 / \mathrm{n} 1048969 \mathrm{z}$

18. Yu, J., Grossiord, N., Koning, C.E., Loos, J.: Controlling the dispersion of multi-wall carbon nanotubes in aqueous surfactant solution. Carbon N. Y. 45, 150 (2007)

19. Vaisman, L., Wagner, D.H., Vaisman, L., Wagner, H.D., Marom, G.: The role of surfactants in dispersion of carbon nanotubes the role of surfactants in dispersion of carbon nanotubes. Adv. Colloid Interface Sci. 128, 37-46 (2015). doi:10.1016/j.cis.2006.11.007

20. Geng, Y., Liu, M.Y., Li, J., Shi, X.M., Kim, J.K.: Composites : part a effects of surfactant treatment on mechanical and electrical properties of CNT/epoxy nanocomposites. Compos. Part A 39, 1876-1883 (2008). doi:10.1016/j.compositesa.2008.09.009

21. Dreyer, D.R., Park, S., Bielawski, C.W., Ruoff, R.S.: The chemistry of graphene oxide. Chem. Soc. Rev. 39, 228-240 (2010). doi:10.1039/b917103g

22. Li, J., Vaisman, L., Marom, G., Kim, J.K.: Br treated graphite nanoplatelets for improved electrical conductivity of polymer composites. Carbon N. Y. 45, 744-750 (2007). doi:10.1016/j.carbon.2006.11.031

23. Li, J., Sham, M.L., Kim, J.K., Marom, G.: Morphology and properties of UV/ozone treated graphite nanoplatelet/epoxy nanocomposites. Compos. Sci. Technol. 67, 296-305 (2007). doi:10.1016/j. compscitech.2006.08.009

24. Ma, P.-C., Mo, S.-Y., Tang, B.-Z., Kim, J.-K.: Dispersion, interfacial interaction and Re- agglomeration of functionalized carbon nanotubes in epoxy composites. Carbon N. Y. 48, 1824-1834 (2010). doi:10.1016/j. carbon.2010.01.028 
25. Prolongo, S.G., Meliton, B.G., Del Rosario, G., Ureña, A.: Simultaneous dispersion and alignment of carbon nanotubes in epoxy resin through chronoamperometry. Carbon N. Y. 50, 5489-5497 (2012). doi:10.1016/j. carbon.2012.07.037

26. R. Sellak, Elaboration et caracterisation d'une resine thermodurcissable conductrice, du Maine, 2013

27. Xie, X.L., Mai, Y.W., Zhou, X.P.: Dispersion and alignment of carbon nanotubes in polymer matrix: a review. Mater. Sci. Eng. R Reports. 49, 89-112 (2005). doi:10.1016/j.mser.2005.04.002

28. Huang, Y.Y., Terentjev, E.M.: Dispersion of carbon nanotubes: mixing, sonication, stabilization, and composite properties. Polymers (Basel) 4, 275-295 (2012). doi:10.3390/polym4010275

29. Kostagiannakopoulou, C., Loutas, T.H., Sotiriadis, G., Markou, A., Kostopoulos, V.: On the interlaminar fracture toughness of carbon fiber composites enhanced with graphene nano-species. Compos. Sci. Technol.

118, 217-225 (2015). doi:10.1016/j.compscitech.2015.08.017

30. P. Nordin, Graphene de-icing/anti-icing and lightning strike protection, 2015.

31. Cheng, Q., Wang, J., Jiang, K., Li, Q., Fan, S.: Fabrication and properties of aligned multiwalled carbon nanotube-reinforced epoxy composites. J. Mater. Res. 23, 2975-2983 (2008). doi:10.1557/JMR.2008.0356

32. Cooper, C.A., Cooper, C.A., Ravich, D., Ravich, D., Lips, D., Lips, D., Mayer, J., Mayer, J., Wagner, H.D.: Wagner, distribution and alignment of carbon nanotubes and nano brils in a polymer matrix. Compos. Sci. Technol. 62, 1105-1112 (2008)

33. Hou, X., et al.: Stretching-induced orientation to improve mechanical properties of electrospun pan nanocomposites QUT ePrints. Mod Phys B. 22, 5913-5918 (2008)

34. McCann, J.T., Marquez, M., Xia, Y.: Highly porous fibers by electrospinning into a cryogenic liquid. J. Am. Chem. Soc. 128, 1436-1437 (2006). doi:10.1021/ja056810y

35. Goh, P.S., Ismail, A.F., Ng, B.C.: Directional alignment of carbon nanotubes in polymer matrices: contemporary approaches and future advances. Compos. Part A Appl. Sci. Manuf. 56, 103-126 (2014). doi:10.1016/j. compositesa.2013.10.001

36. Wang, M., Anoshkin, I.V., Nasibulin, A.G., Korhonen, J.T., Seitsonen, J., Pere, J., Kauppinen, E.I., R.H.A, Ras, O.: Ikkala, modifying native nanocellulose aerogels with carbon nanotubes for mechanoresponsive conductivity and pressure sensing. Adv. Mater. (2013). doi:10.1002/adma.201300256

37. Wu, S., Ladani, R.B., Zhang, J., Bafekrpour, E., Ghorbani, K., Mouritz, A.P., Kinloch, A.J., Wang, C.H.: Aligning multilayer graphene flakes with an external electric field to improve multifunctional properties of epoxy nanocomposites. Carbon N. Y. 94, 607-618 (2015). doi:10.1016/j.carbon.2015.07.026

38. Ladani, R.B., Ravindran, A.R., Wu, S., Pingkarawat, K., Kinloch, A.J., Mouritz, A.P., Ritchie, R.O., Wang, C.H.: Multi-scale toughening of fi bre composites using carbon nano fi bres and z-pins. Compos. Sci. Technol. 131, 98-109 (2016). doi:10.1016/j.compscitech.2016.06.005

39. Li, J., Kim, J.: Percolation threshold of conducting polymer composites containing graphite nanoparticles. Compos. Sci. Technol. 67, 2114-2220 (2007)

40. Rastogi, R., Kaushal, R., Tripathi, S.K., Sharma, A.L., Kaur, I., Bharadwaj, L.M.: Comparative study of carbon nanotube dispersion using surfactants. J. Colloid Interface Sci. 328, 421-428 (2008). doi:10.1016/j. jcis.2008.09.015

41. Matarredona, O., Rhoads, H., Li, Z., Harwell, J.H., Balzano, L., Resasco, D.E.: Dispersion of single-walled carbon nanotubes in aqueous solutions of the anionic surfactant NaDDBS. J. Phys. Chem. B 107, 1335713367 (2003). doi:10.1021/jp0365099

42. Bergey, D.M., Johnson, A.T.: High weight fraction surfactant solubilization of single-wall carbon nanotubes in water. Nano Lett. 3, 269-273 (2003)

43. Bai, Y., Lin, D., Wu, F., Wang, Z., Xing, B.: Adsorption of triton X-series surfactants and its role in stabilizing multi-walled carbon nanotube suspensions. Chemosphere 79, 362-367 (2010). doi:10.1016/j. chemosphere.2010.02.023

44. Gong, X., Liu, J., Baskaran, S., Voise, R.D., Young, J.S.: Surfactant-assisted processing of carbon nanotube/ polymer composites. Chem. Mater. 12, 1049-1052 (2000). doi:10.1021/cm9906396

45. Reed, C.W.: The chemistry and physics of the interface region and functionalization. Dielectr. Polym. Nanocomposites (2010). doi:10.1007/978-1-4419-1591-7_4

46. Prolongo, S.G., et al.: Advantages and disadvantages of the addition of graphene nanoplatelets to epoxy resins. Eur. Polym. J. 61, 206-214 (2014)

47. Sharma, R., Baik, J.H., Perera, C.J., Strano, M.S.: Anomalously large reactivity of single graphene layers and edges toward electron transfer chemistries. Nano Lett. 10, 398-405 (2010). doi:10.1021/n1902741x

48. Barrau, S., Demont, P., Peigney, A., Laurent, C., Lacabanne, C.: DC and AC conductivity of carbon nanotubes-polyepoxy composites. Macromolecules 36, 5187-5194 (2003)

49. Cui, S., Canet, R., Derre, A., Couzi, M., Delhaes, P.: C haracterization of multiwall carbon nanotubes and in uence of surfactant in the nanocomposite processing. Carbon N. Y. 41, 797-809 (2003)

50. Jin, Y., Sun, T., Do, H., Hwa, J.: Electrical conductivity of chemically modified multiwalled carbon nanotube/epoxy composites. Carbon N. Y. 43, 23-30 (2005). doi:10.1016/j.carbon.2004.08.015 
51. Michelis, P., Vlachopoulos, J.: Complete CNT disentanglement-dispersion-functionalisation in a pulsating micro-structured reactor. Chem. Eng, Sci (2013). doi:10.1016/j.ces.2012.12.003

52. Nadiv, R., Shtein, M., Buzaglo, M., Peretz-Damari, S., Kovalchuk, A., Wang, T., Tour, J.M., Regev, O.: Graphene nanoribbon-polymer composites: the critical role of edge functionalization. Carbon N. Y. 99, 444 450 (2016). doi:10.1016/j.carbon.2015.12.039

53. Ahmadi-Moghadam, B., Sharafimasooleh, M., Shadlou, S., Taheri, F.: Functionalization of graphene nanoplatelets on the mechanical response of graphene/epoxy composites. Mater. Des. 66, 142-149 (2015)

54. Chiu, P.W., Duesberg, G.S., Dettlaff-Weglikowska, U., Roth, S.: Interconnection of carbon nanotubes by chemical functionalization. Appl. Phys. Lett. 80, 3811-3813 (2002). doi:10.1063/1.1480487

55. Hashemi, R.: On the overall viscoelastic behavior of graphene/polymer nanocomposites with imperfect interface. Int. J. Eng. Sci. 105, 38-55 (2016). doi:10.1016/j.ijengsci.2016.04.006

56. Gojny, F.H.: Carbon nanotube-reinforced epoxy-composites : enhanced stiffness and fracture toughness at low nanotube content. Compos. Sci. Technol. 64, 2363-2371 (2004). doi:10.1016/j. compscitech.2004.04.002

57. Ma, P.C., Siddiqui, N.A., Marom, G., Kim, J.K.: Dispersion and functionalization of carbon nanotubes for polymer-based nanocomposites: a review. Compos. Part A Appl. Sci. Manuf. 41, 1345-1367 (2010). doi:10.1016/j.compositesa.2010.07.003

58. Sengupta, R., Bhattacharya, M.: A review on the mechanical and electrical properties of graphite and modified graphite reinforced polymer composites. Prog. Polym. 36, 638-670 (2010)

59. Prolongo, S.G., Jimenez-Suarez, A., Moriche, R., Ureña, A.: In-situ processing of epoxy composites reinforced with graphene nanoplatelets. Compos. Sci. Technol. 86, 185-191 (2013). doi:10.1016/j. compscitech.2013.06.020

60. Hu, N., Karube, Y., Arai, M., Watanabe, T., Yan, C., Li, Y., Liu, Y., Fukunaga, H.: Investigation on sensitivity of a polymer/carbon nanotube composite strain sensor. Carbon N. Y. 48, 680-687 (2010). doi:10.1016/j. carbon.2009.10.012

61. Larijani, M.M., Khamse, E.J., Asadollahi, Z., Asadi, M.: Effect of aligned carbon nanotubes on electrical conductivity behaviour in polycarbonate matrix. Bull. Mater. Sci. 35, 305-311 (2012). doi:10.1007/s12034012-0299-1

62. Sharma, A., Tripathi, B., Vijay, Y.K.: Dramatic Improvement in properties of magnetically aligned CNT/ polymer nanocomposites. J. Memb. Sci. 361, 89-95 (2010). doi:10.1016/j.memsci.2010.06.005

63. Kimura, T., Ago, H., Tobita, M., Ohshima, S., Kyotani, M., Yumura, M.: Polymer composites of carbon nanotubes aligned by a magnetic field. Adv. Mater. 14, 1380-1383 (2002). doi:10.1002/1521-4095 (20021002)14:193.0.CO;2-V

64. Bhardwaj, S., Cepek, C., Giorcelli, M., Tagliaferro, A.: Alignments of carbon nanotubes in polymer matrix: a raman perspective. Int. J. Polym. Anal. Charact. (2012). doi:10.1080/1023666X.2012.704559

65. Zhan, Y., Meng, F., Yang, X., Zhao, R., Liu, X.: Solvothermal synthesis and characterization of functionalized graphene sheets (FGSs)/magnetite hybrids. Mater. Sci. Eng. B 176, 1333-1339 (2011). doi:10.1016/j. mseb.2011.07.023

66. Shi, D., He, P., Zhao, P., Guo, F.F., Wang, F., Huth, C., Chaud, X., Bud'Ko, S.L., Lian, J.: Magnetic alignment of $\mathrm{Ni} / \mathrm{Co}-$ coated carbon nanotubes in polystyrene composites. Compos. Part B Eng 42, 15321538 (2011). doi:10.1016/j.compositesb.2011.04.014

67. Jia, B., Gao, L., Sun, J.: Self-assembly of magnetite beads along multiwalled carbon nanotubes via a simple hydrothermal process. Carbon N. Y. 45, 1476-1481 (2007). doi:10.1016/j.carbon.2007.03.025

68. Prolongo, S.G., Meliton, B.G., Del Rosario, G., Ureña, A.: New alignment procedure of magnetite-CNT hybrid nanofillers on epoxy bulk resin with permanent magnets. Compos. Part B Eng. 46, 166-172 (2013). doi:10.1016/j.compositesb.2012.10.002

69. Abdalla, M., Dean, D., Theodore, M., Fielding, J., Nyairo, E., Price, G.: Magnetically processed carbon nanotube/epoxy nanocomposites: morphology, thermal, and mechanical properties. Polymer (Guildf) 51, 1614-1620 (2010). doi:10.1016/j.polymer.2009.05.059

70. Martin, C.A., Sandler, J.K.W., Windle, A.H., Schwarz, M.K., Bauhofer, W., Schulte, K., Shaffer, M.S.P.: Electric field-induced aligned multi-wall carbon nanotube networks in epoxy composites. Polymer (Guildf) 46, 877-886 (2005). doi:10.1016/j.polymer.2004.11.081

71. Li, W., Dichiara, A., Zha, J., Su, Z., Bai, J.: On improvement of mechanical and thermo-mechanical properties of glass fabric/epoxy composites by incorporating CNT-A12O3 hybrids. Compos. Sci. Technol. 103, 36-43 (2014). doi:10.1016/j.compscitech.2014.08.016

72. Renteria, J., Legedza, S., Salgado, R., Balandin, M.P., Ramirez, S., Saadah, M., Kargar, F., Balandin, A.A.: Magnetically-functionalized self-aligning graphene fillers for high-efficiency thermal management applications. Mater. Des. 88, 214-221 (2015). doi:10.1016/j.matdes.2015.08.135 
73. Piao, G., Kimura, F., Takahashi, T., Moritani, Y., Awano, H., Nimori, S., Tsuda, K., Yonetake, K., Kimura, T.: Alignment and micropatterning of carbon nanotubes in polymer composites using modulated magnetic field. Polym. J. 39, 589-592 (2007). doi:10.1295/polymj.PJ2006191

74. Sepioni, M.: Magnetic properties of graphene. University of Manchester, Manchester (2012). doi:10.1002/smll

75. Wang, F., Drzal, L.T., Qin, Y., Huang, Z.: Mechanical properties and thermal conductivity of graphene nanoplatelet/epoxy composites. J. Mater. Sci. 50, 1082-1093 (2014). doi:10.1007/s10853-014-8665-6

76. King, J.A., Klimek, D.R., Miskioglu, I., Odegard, G.M.: Mechanical properties of graphene nanoplatelet/ epoxy composites. J. Appl. Polym. Sci. (2012). doi:10.1002/app.38645

77. Manta, A., Gresil, M., Soutis, C.: Multi-scale finite element analysis of graphene/polymer nanocomposites: electrical perfomance. Appl. Compos, Mater (2016)

78. Sun, X., Ramesh, P., Itkis, M.E., Bekyarova, E., Haddon, R.C.: Dependence of the thermal conductivity of two-dimensional graphite nanoplatelet-based composites on the nanoparticle size distribution. J. Phys. Condens. Matter 22, 334216 (2010). doi:10.1088/0953-8984/22/33/334216 